\title{
Ultrasonic/Sonic Driller/Corer (USDC) as a Sampler for Planetary Exploration
}

\author{
Yoseph Bar-Cohen, Stewart Sherrit, Benjamin P. Dolgin, \\ Nathan Bridges, Xiaoqi Bao, Zensheu Chang, \\ Albert Yen, Ronald S. Saunders \\ Jet Propulsion Laboratory/California Institute of Technology \\ 4800 Oak Grove Drive. M/S 82-105 \\ Pasadena, CA 91109-8099 \\ yosi@jpl.nasa.gov, Web: http://ndeaa.jpl.nasa.gov
}

\author{
Dharmendra Pal, Jason Kroh, Tom Peterson \\ Cybersonics Inc. \\ 5368 Kuhl Road \\ Erie, PA 16510 \\ (814) 899-4220 \\ cyber@cybersonicsinc.com
}

Abstract-Future NASA exploration missions to Mars, Europa, Titan, comets, and asteroids will perform sampling, in-situ analysis and possibly the return of material to Earth for further tests. One of the major limitations of sampling in low gravity environments is that conventional drills need high axial force. An ultrasonic/sonic driller/corer (USDC) mechanism was developed to address these and other limitations of existing drilling techniques. The USDC is based on an ultrasonic horn that is driven by a piezoelectric stack. The horn drives a free-mass, which resonates, between the horn and drill stem. Tests have shown that this device addresses some of the key challenges to the NASA objective of planetary in-situ sampling and analysis. The USDC is lightweight ( $450 \mathrm{~g})$, requires low pre-load $(<5 \mathrm{~N})$ and can be driven at low power $(5 \mathrm{~W})$. The device was operated from such robotic platforms as the Sojourner rover and the FIDO robotic arm and it has been shown to drill various rocks including granite, diorite, basalt and limestone. The drill can potentially operate at high and low temperatures and does not require sharpening. Although the drill is driven electrically at $20 \mathrm{kHz}$, a substantial sub-harmonic acoustic component is found that is crucial to drilling performance. Models that explain this low frequency coupling in the horn, free-mass, drill stem and rock are presented. Efforts are currently underway to integrate the models and experimentally corroborate the predictions.

\section{TABLE OF CONTENTS}

1. INTRODUCTION

2. MODELING

2.1 HORN TIP-FREE-MASS INTERACTION

2.2 FREE-MASS-DRILL STEM INTERACTION

2.3 BIT-ROCK INTERACTION

3. ROCK SAMPLING AND EVALUATION

4. OPERATION FROM VARIOUS PLATFORMS

5. CONCLUSIONS

\section{INTRODUCTION}

Efficient drilling and coring of rock materials has a great significance to the NASA planetary program where a sample needs to be cored, in-situ analyzed, and possibly returned to Earth. Other fields can also benefit from an effective drilling, including military, medical operations, construction, geology, sport (e.g., hiking), and games. Existing drilling techniques are limited by the need for large axial forces, high power consumption, and heavy mechanisms. In addition, conventional drillers are subject to drill bit jamming, breaking, dulling and are difficult to use in non-horizontal or hard surfaces drilling. Also, the drilling process is hampered by the accumulation of drilling-debris in the drilled area. The life of coring bits is markedly reduced by the breakdown of the binder that holds the abrasive material on the bit surface. The ability of the new ultrasonic/sonic driller/corer to operate with minimum axial load (see Figure 1) offers important enabling technology. The authors' efforts are directed towards understanding the mechanism and analytically modeling its operation thereby optimizing its performance.

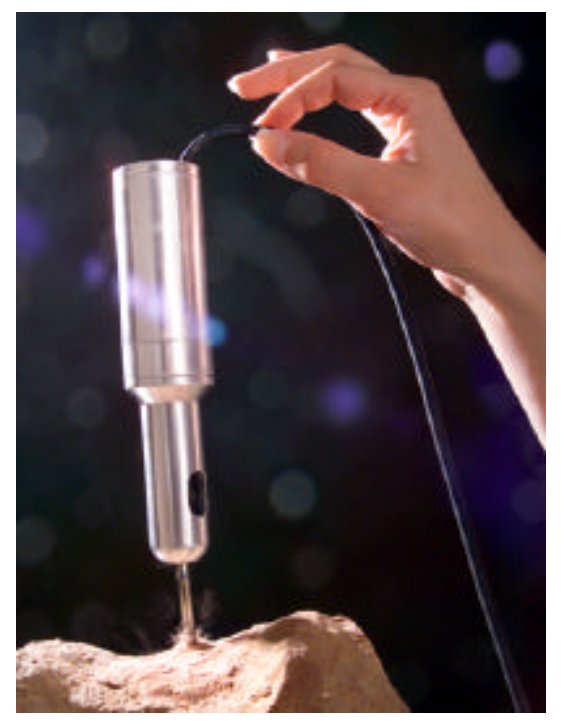

Fig. 1. A photographic view of the USDC held from its cable while coring sandstone. The minimal need for axial force is easily seen.

To establish the required analytical model simultaneous theoretical and experimental studies have been launched and data were collected to assist in understanding the mechanism. The experimental setup consists of a USDC 
that is attached to a test rig as shown in Figure 2 both schematically and photographically. The device consists of three main parts: an ultrasonic actuator, a free-mass and a drill stem. The analysis of the actuator circuit was based on the equivalent circuit of piezoelectric and acoustic elements as discussed by Mason [1,2] as was reported previously [3]. The horn was found to have a resonance at $21.5 \mathrm{kHz}$ and the free tip velocity at resonance was determined to be linear with respect to the applied voltage and ranged from 1 to 10 $\mathrm{m} / \mathrm{s}$ depending on the acoustic load.
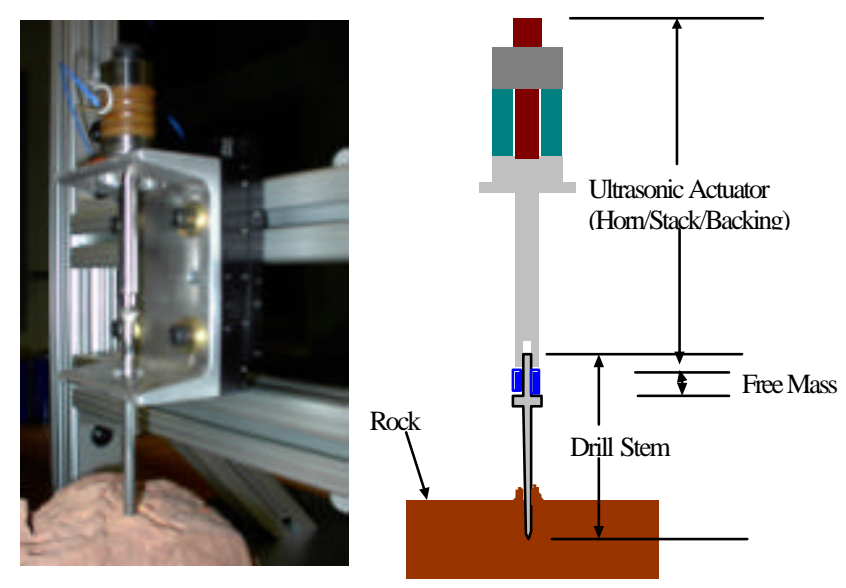

Fig. 2. Photograph and Schematic of the Ultrasonic/Sonic Driller/Corer.

The vibrations of the horn tip excite the free-mass, which resonates between the horn tip and the top of the drill stem at frequency of the order of $1000 \mathrm{~Hz}$. Acoustic energy in the free-mass resonator is transferred to the top of the drill stem and propagates to the bit/rock interface, where the rock is excited past its ultimate strain, and fractures. In order to determine the critical issues related to the control and optimization of the drill, initial modeling of the acoustic interaction at the various interfaces of the drill is presented. The three interfaces that were studied are: (1) the interaction of the horn tip with the free-mass, (2) the interaction of the free-mass with the drill stem, and (3) the interaction of the base of the drill stem (bit) with the rock.

Generally, the USDC is highly tolerant of changes in its operating environment. Because is it driven by piezoelectric ceramics the USDC can be designed to operate at a wide range of temperatures including those expected on Mars $\left(-60^{\circ} \mathrm{C}\right)$ and Venus $\left(+450{ }^{\circ} \mathrm{C}\right)$. The operation in low temperatures was successfully demonstrated while the high temperature potential is currently being investigated. The coring bit creates a hole that is slightly larger than the drill bit diameter. This latter reduces the chances of bit jamming if the integrity of the hole is maintained, thus avoiding another problem associated with conventional drilling. The ultrasonic bit need not be sharp. Thus, it does not have problems with bit dulling and wear. USDC is a novel device, not a conventional ultrasonic drill. Although the drill is driven electrically at $20 \mathrm{kHz}$ a substantial sub-harmonic acoustic component is found that is crucial to drilling performance. An analytical model has been developed to explain this low frequency coupling interaction in the horn, free-mass behavior, drill stem, and rock.

\section{MODELING}

In order to proceed with the modeling, the velocity/ displacement of the horn tip during operation is required. Since the velocity/displacement depends on the acoustic load, a reasonable estimate of the velocity range can determined by calculating the horn tip velocity for the case where it is free and when the acoustic impedance is perfectly matched to produce maximum power in the acoustic load. The velocity of the tip as a function of the applied peak AC voltage is shown in Figure 3 for the two cases. The reasons for using the case where the acoustic load is ideal rather than clamped are twofold. Firstly, the acoustic power transferred to the rock is substantial, which means that the horn is well matched to the rest of the acoustic elements, and secondly, in order for the device to operate efficiently a gap must be present, which means that for a substantial portion of the time the tip is not in contact with the free-mass. For a peak AC voltage of 200 Volts on the drill the velocity is found to range between $1.25 \mathrm{~m} / \mathrm{s}$ for the ideal load and $13.6 \mathrm{~m} / \mathrm{s}$ for the free tip. The modeling of the interaction of the free-mass can now be determined since the range of speeds of the horn tip has been set.

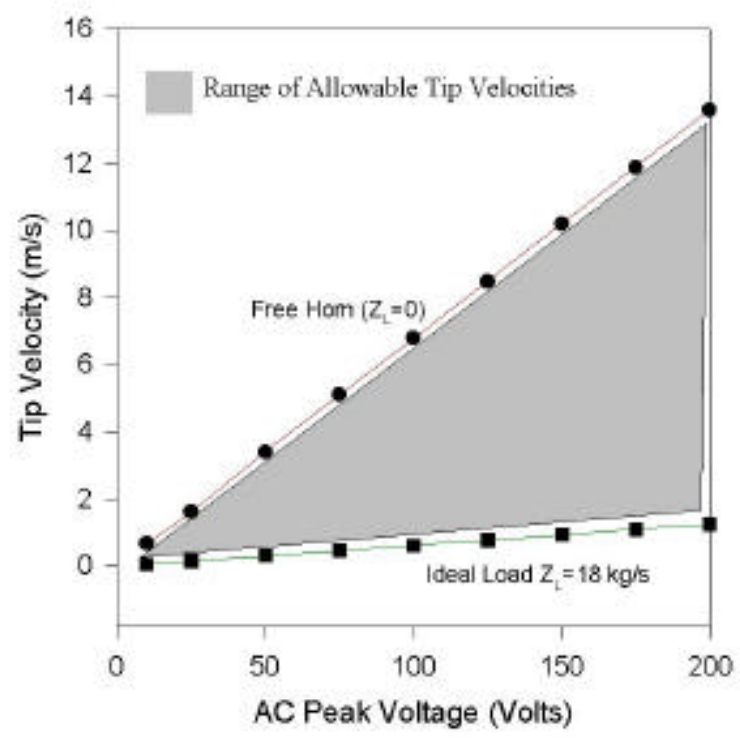

Fig. 3. The range of speed for the horn tip for an ideal load and for the unloaded horn.

\subsection{Horn Tip-Free-mass Interaction}

The free-mass is driven by the horn tip, which vibrates at resonance frequency. A schematic of the model is shown in Figure 4. The tip displacement is harmonic and is describe by

$$
\mathrm{u}=\mathrm{u}_{0} \cos (\omega \mathrm{t}+\theta) \text {. }
$$


The velocity of the horn tip is found by taking the time derivative of the displacement and is

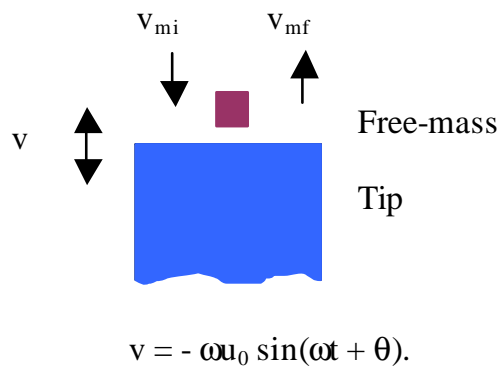

Fig. 4. A schematic of the free-mass horn tip interaction model.

Assuming the energy loss and time duration of the impact is negligible and the mass of the horn is much larger than the free-mass, we find, using conservation of momentum and energy, that

$$
\mathrm{v}_{\mathrm{mf}}=\mathrm{v}_{\mathrm{mi}}+2 \mathrm{v}
$$

where $\mathrm{v}_{\mathrm{mi}}$ is the free-mass velocity prior to interaction with the horn and $\mathrm{v}_{\mathrm{mf}}$ is the free-mass velocity after interaction with the horn. A computer simulation model, which traces the positions of the free-mass until it leaves the tip vibration range $\left(2 \mathbf{u}_{0}\right)$, was written. The routine calculates the free-mass speed after interaction with the horn. By keeping track of the free-mass velocity, the routine allowed us to explore this driving mechanism as a function of the horn position as the free-mass crosses the plane of full extension (phase). The speed of the free-mass as it leaves the interaction region versus position of the horn (position of tip represented as a phase angle $\mathrm{t}=0$ at max displacement) is shown in Figure 5 for various ratios of $\mathrm{v}_{\mathrm{mi}} / \mathrm{v}$.

For all cases there is a net increase in the free-mass velocity after interaction with the horn when averaged over phase. The computer model traces the movements of the free-mass and the horn. The movement of the horn is due to the reaction force on the horn tip and the force of gravity. In the calculation the mass of the free-mass is set to 1 gram, the tip velocity amplitude is set to $1.26 \mathrm{~m} / \mathrm{s}$ corresponding to displacement amplitude of $10 \mu \mathrm{m}$. The horn mass was $800 \mathrm{~g}$. The energy loss of the free-mass in each round trip is set as $75 \%$. The simulation results shown in Figure 6 represent the ratio of the free-mass velocity to the maximum horn tip velocity as a function of time. In addition, the data was used to calculate the motion of the horn. The results show the random characteristics of the movement of the body of the horn. Figure 7 shows the horn tip position as a function of time. The motion is due to the impact interaction with the free-mass.
The size, frequency and randomness of the jumps are confirmed by experimental observations of the horn tip by a high-speed camera (Figure 8). 
Fig. 5. The free-mass velocity after interaction with the horn for $\mathrm{v}_{\mathrm{mi}}=0.2,1,2,4 \mathrm{v}$. 


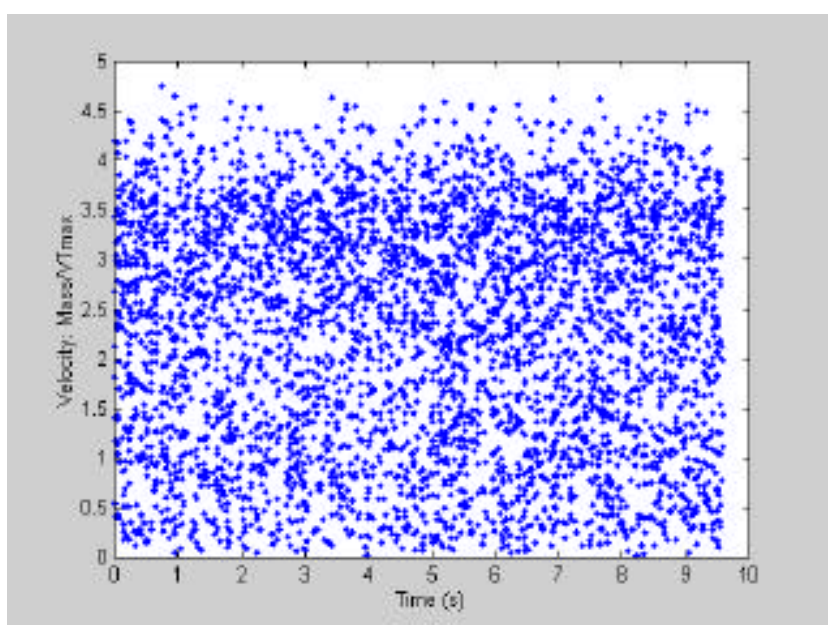

Fig. 6. The free-mass velocity/maximum tip velocity as a function of time.

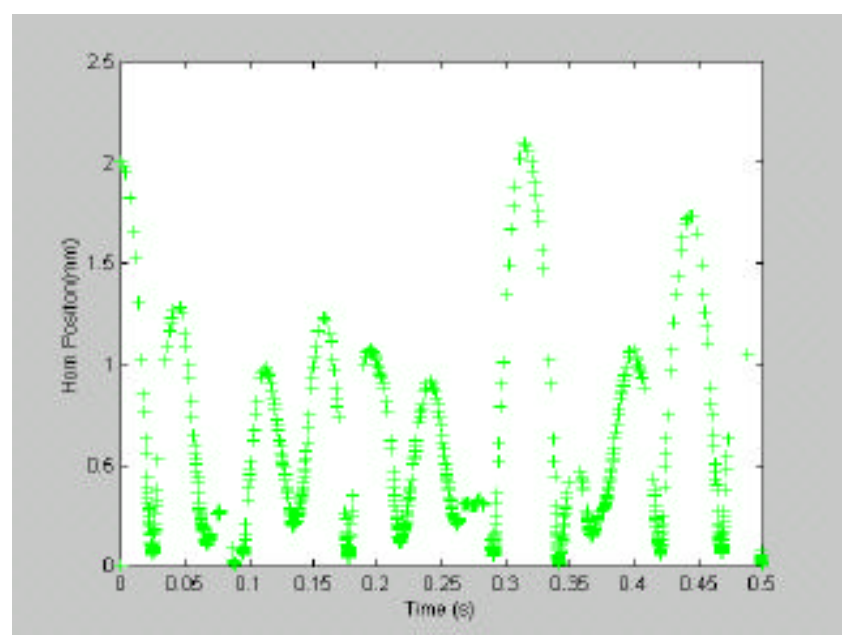

Fig. 7. The horn position as a function of time determined from model.

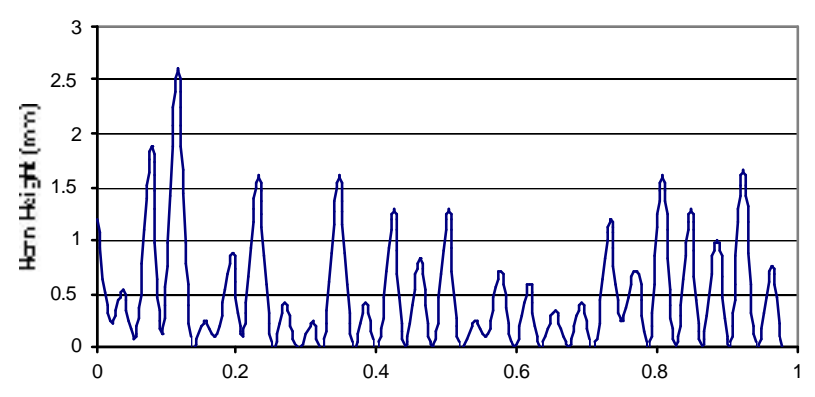

Fig. 8. The horn position as a function of time determined from high-s peed camera.

\subsection{Free-mass-Drill Stem Interaction}

The free-mass impacts the top of the drill stem and creates a stress wave that propagates to the bit end. A finite element model was utilized to investigate the impact and resultant stress wave. Figure 9 shows results for the case where a cylindrical steel stem/bit of diameter $3.0 \mathrm{~mm}$ and length $100 \mathrm{~mm}$ with a concentric top cap of diameter $12 \mathrm{~mm}$ and length $6 \mathrm{~mm}$. The impact used in the model is that of a freemass of $2 \mathrm{~g}$ with speed $1 \mathrm{~m} / \mathrm{s}$. The free-mass has a curved surface at the contact area with a curvature of $10 \mathrm{~mm}$. The free-mass is assumed to be rigid and the bit end of the drill stem is clamped. Figure 9 shows the displacement of the surface of the free-mass and the top surface of the drill stem during impact as a function of time. Figure 10 shows the resultant stress wave as a function of time at the bit end of the drill stem.

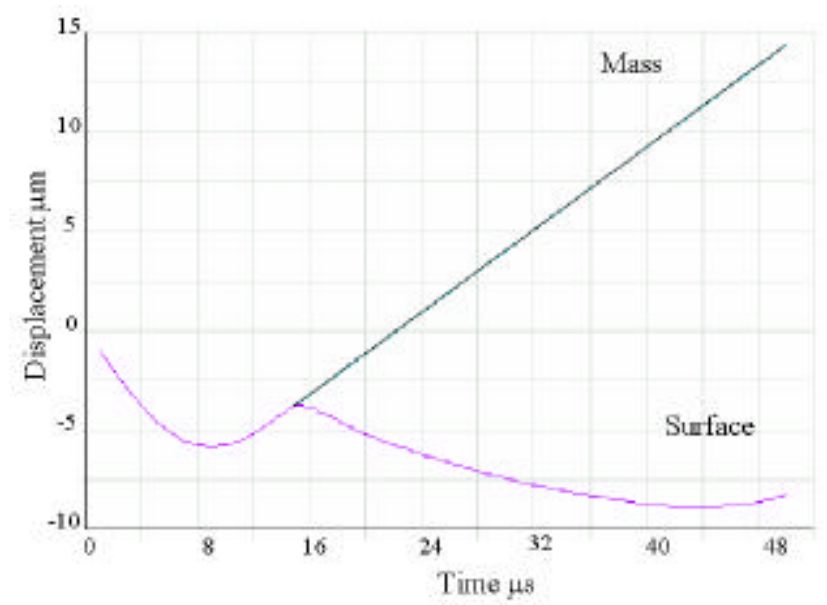

Fig. 9. The displacement as a function of time of the freemass and the top surface of the drill stem after impact.

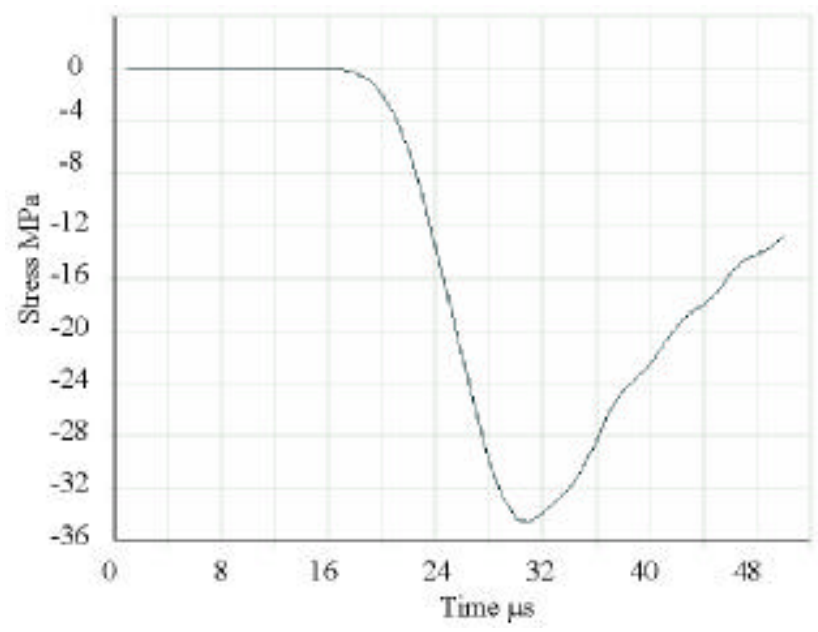

Fig. 10. The stress as a function of time of the free-mass and the top surface of the drill stem after impact. 


\subsection{Bit-Rock Interaction}

To better understand the fracture of rocks under impact loading from a drill or a corer, a finite element model was developed using ANSYS. For the purpose of simplifying the problem, the rock is modeled as a circular cylinder with bottom surface fixed and the drill/corer impacts at the center of the top surface. This simplification makes the problem axisymmetric. By using the axi-symmetric elements available in ANSYS, the original three-dimensional problem is now reduced to two-dimensions. The element size is made very fine near the drill/corer bit, and becomes coarser and coarser as it goes further away from the bit. Figure 11 shows a typical mesh for the problem outlined above.

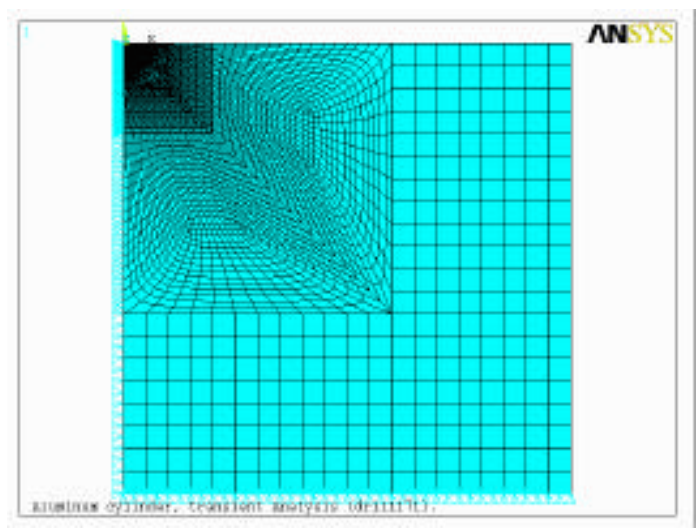

Fig. 11. The mesh used to solve the bit rock interaction.

Preliminary results were derived by assuming that the circular cylinder is made of isotropic material with a Young's modulus of $10 \mathrm{GPa}$ and Poisson's ratio of 0.3 . The impact loading from the drill has a peak value of $0.1 \mathrm{GPa}$ and duration of $2.5 \mu \mathrm{sec}$, as shown in Figure 10.

Contour maps of the maximum principal strain were plotted and used as indication of fracture of rocks. It also shows how the elastic waves propagate in the rock. Figures 12 show the contour maps for the cylinder for drilling and coring, respectively. The drill bit is $3 \mathrm{~mm}$ in diameter. The corer has an inner diameter of $2 \mathrm{~mm}$ and an outer diameter of $3 \mathrm{~mm}$. The results shown in this Figure qualitatively illustrate the rock fracture characteristics that are induced by the ultrasonic drilling or coring. From Figure 12 we find that the highest principal strain occurs at the edge of the drill bit. Also, the highest principal strain appears at both the outer and inner edge of the corer. It implies that the fracture is likely going to happen at the edge, which is confirmed by viewing the high speed filming during drilling. By comparing the various strain profiles in Figure 12, we find that the maximum principal strain under coring is higher than that under drilling, and the area of high principal strain under coring is also larger than that under drilling. It implies that with the same outer diameter and under the same loading, a corer will drill faster than a solid drill of the same diameter.

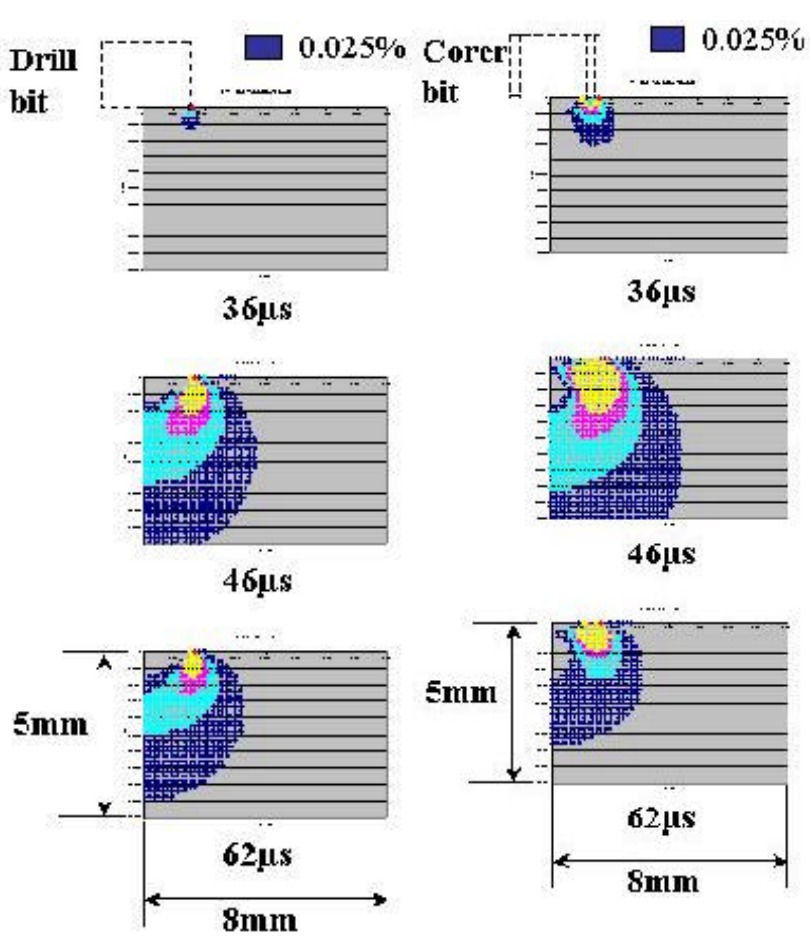

Fig. 12. The principle strain profile at various times after impact of the free-mass on the drill stem for a drilling bit and a coring bit.

This is confirmed by experiments and the results have shown how drilling and coring can be achieved with little or no pre-load. The next challenge is to integrate each of the models to determine the limits and optimization of the USDC.

\section{ROCK SAMPLING AND EVALUATION}

To assure the effective applicability of the USDC to Mars exploration, it is necessary to determine its ability to sample rocks and that the produced tailing will have minimum destruction of its original features. Jointly with JPL geologists, rocks with mechanical properties as expected on Mars were selected. Using the test fixture that was shown in Figure 2, the selected rocks were sampled and the rock tailing was collected for analysis. In parallel drilling performance data was acquired to allow determining the efficiency of the USDC. Using a flat drill bit having 2.85-mm diameter the depth as a function of time was measured and is shown in Figure 13.

This test was conducted using 12-Watts average power (24 Watts peak power- $50 \%$ duty cycle). The power was measured at the input of the electronics and includes the circuitry loss of the driving electronics. For these data the drilling depth as a function of time can be describe by the functional relationship

$$
\mathrm{D}(\mathrm{t})=\mathrm{D}_{0} \mathrm{t}^{\mathrm{n}}
$$


where $D_{0}$ and $n$ are constants that depend on the drill bit geometry and the rock type for a given power level. The value of the constant $n$ is between 0 and 1 . The drill rate can be determined by taking the time derivative and it is

$$
\mathrm{R}(\mathrm{t})=\mathrm{nD}_{0} \mathrm{t}^{\mathrm{n}-1}
$$

The rate is seen to decrease as a function of the time (depth). This saturation is likely due to a combination of causes including the collection of tailings at the face of the bit damping the impact on the rock, viscous drag of the tailings on the drill stem, and non-linear elastic properties of the rock.

In order to determine if the drilling action alters the rock mineralogy four samples of drill tailings from each type were collected from the USDC as they transported from the hole by the ultrasonic mechanism and compared with the powder produced by conventional lab grounding for the same materials. Five different rock samples were chosen for this study including Iron Oxide, 2 Iron Oxyhydroxides samples, Pyroxene and a Carbonate sample. A comparison of the absorbance and the X-ray diffraction for the lab ground and USDC produced tailings is shown in Figures 14 and 15. In general, only minimal differences were observed between the two powder production techniques.

Several bit types were tested to determine the drilling/coring and abrasion rates. Examples of these are shown in Figure 16.

An example of the rock abrasion tools is shown in Figure 17. The letters JPL were carved into the basalt using the tungsten carbide coated sphere in approximately 40 minutes using 12 watts power. The circular area below and to the right was carved using the 2D array of pyramidal bits. When compared with conventional drilling using similar power levels the USDC showed twice the rate for Bishop Tuff rock.
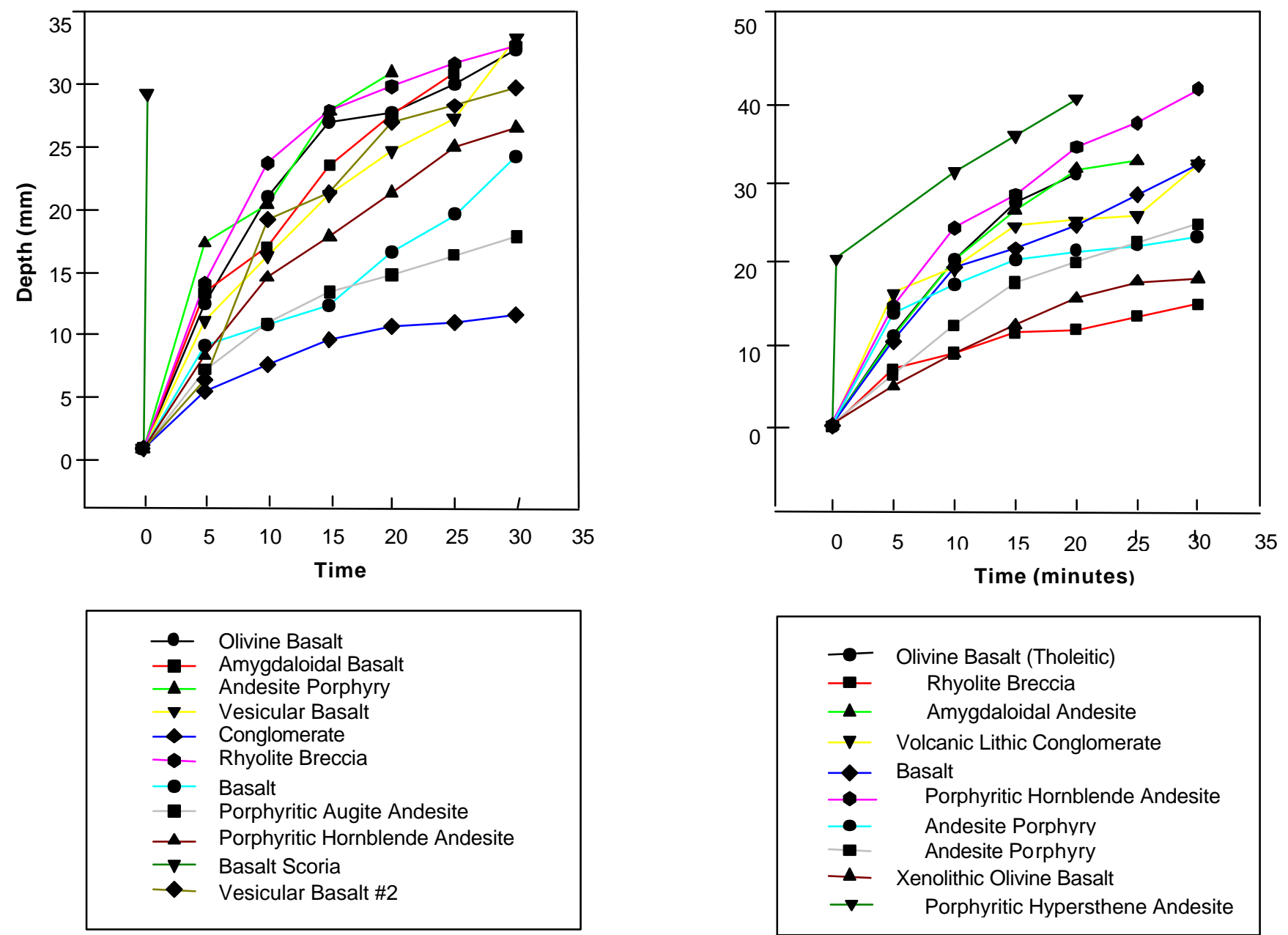

Set 1

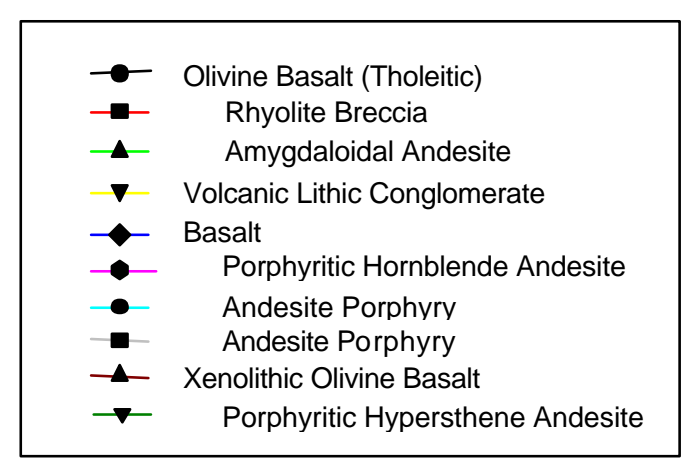

Set 2

Fig. 13. The depth in $\mathrm{mm}$ as a function of time for various rock types. 
2001 IEEE Aerospace Conference on the topic of "Missions, Systems, and Instruments for In Situ Sensing" (Session 2.05). March 10-17, 2001, Big Sky, Montana. http://www.aeroconf.org/.

IEEE copyright $\odot 2001$

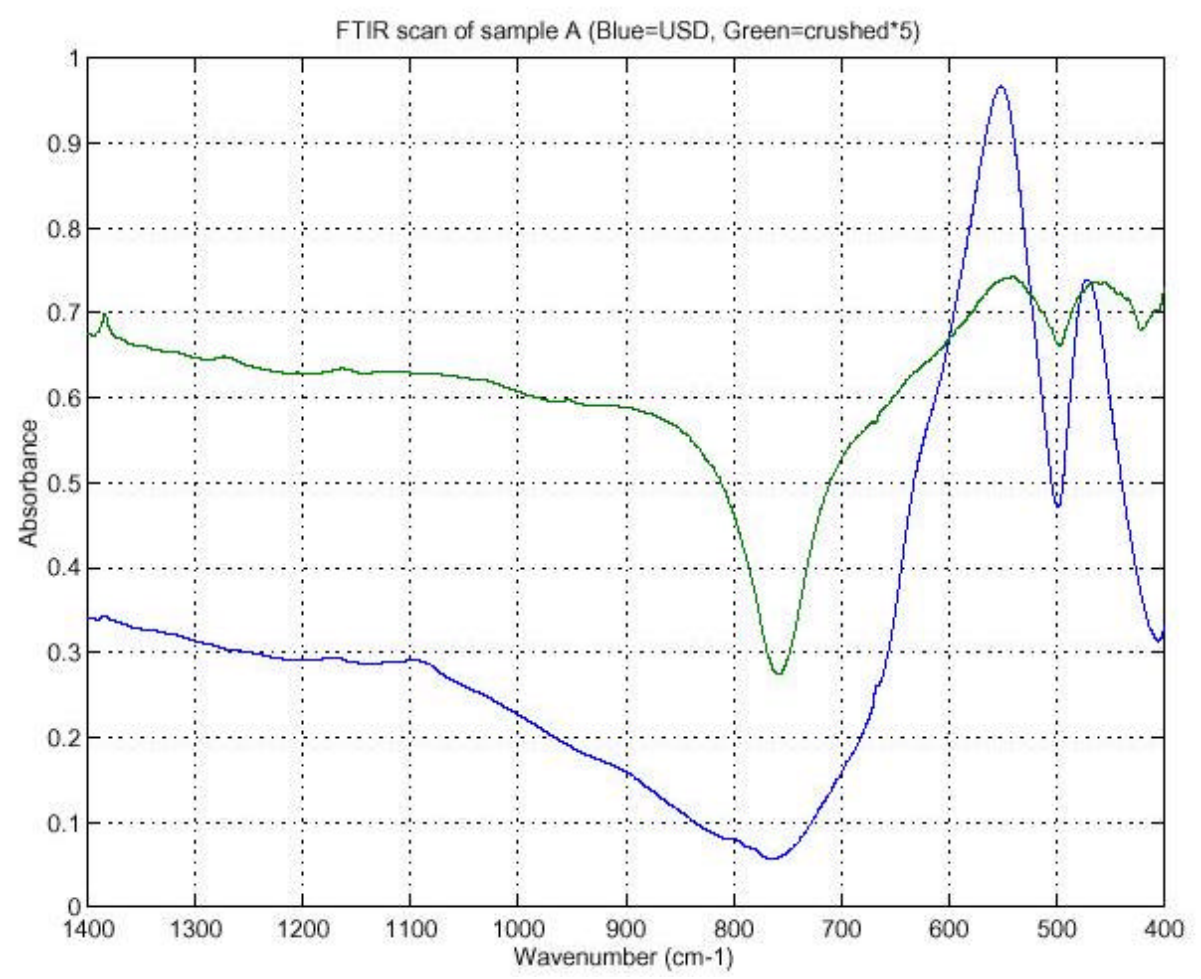

Fig. 14. The FTIR spectra of the lab ground (green line) and the USDC produce powder for a hematite rock sample. Peaks in the spectra are found to overlap.

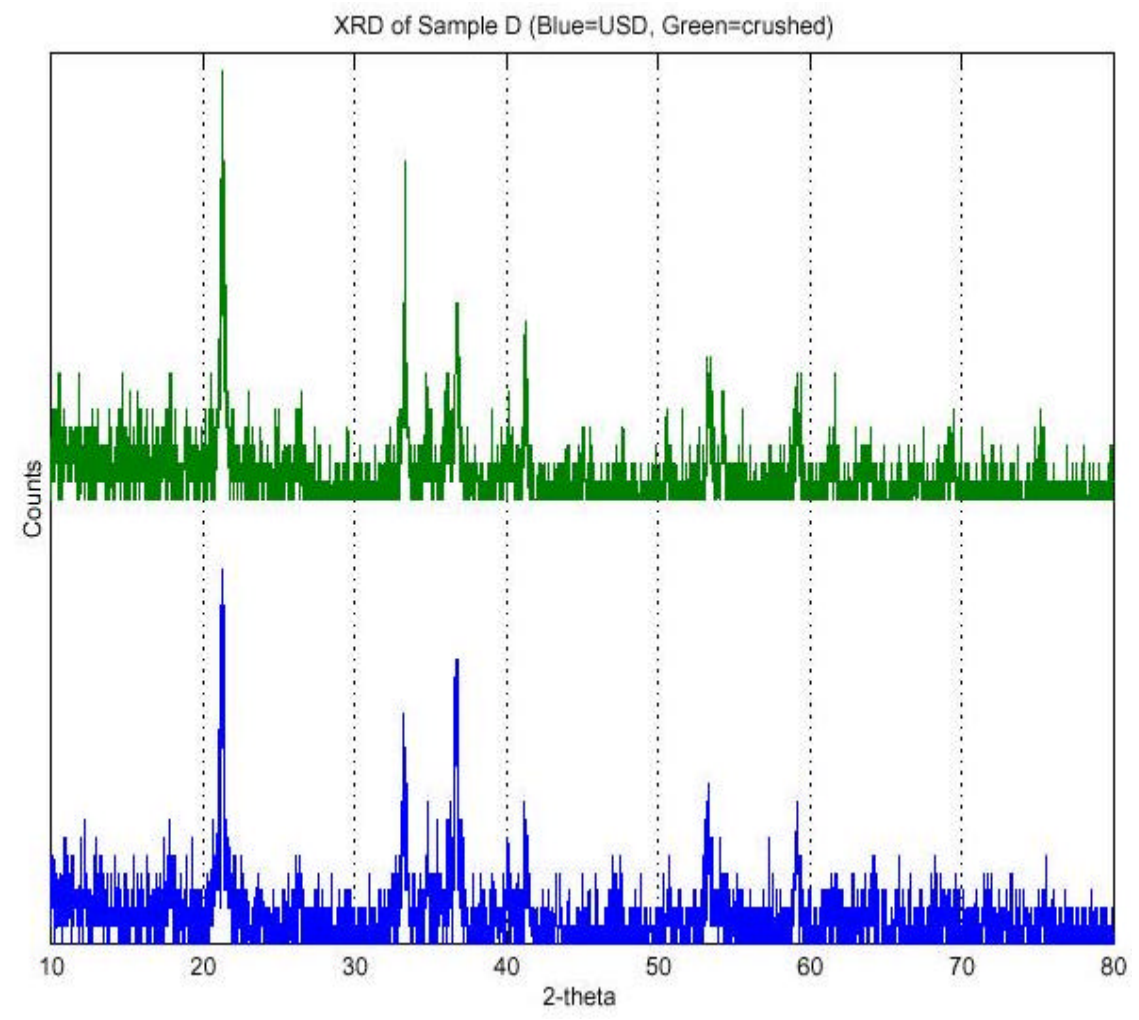


Fig. 15. XRD spectra of the lab ground (green line) and the USDC produce powder for an Iron Oxyhydroxide rock sample. Peaks in the spectra are found to overlap. There is no evidence of conversion to hematite during drilling.

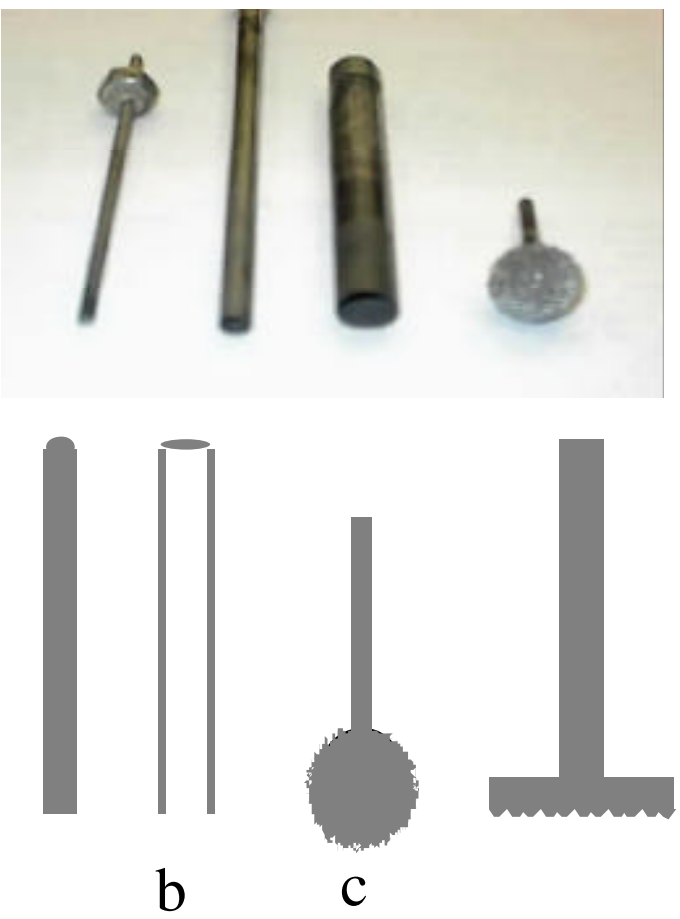

Fig. 16. Photograph and schematic of a variety of bit designs being tested for coring, drilling, and rock abrasion. (a) Tungsten carbide flat-bit drills, (b) stainless coring tubes, (c) tungsten carbide fiber-coated spheres, and (d) an abrasion bit formed by a 2D array of pyramidal bits.

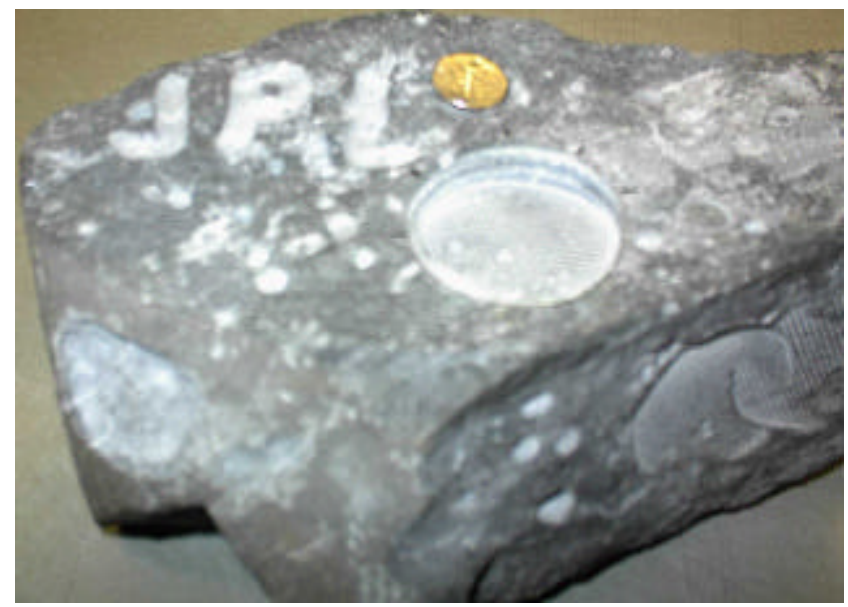

Fig. 17. Basalt rock sample tested using the abrasion tools shown in Fig. 17.

\section{OPERATION FROM VARIOUS PLATFORMS}

Given the low axial force that is needed to drill with the USDC, a series of demonstrations were made to show the feasibility of this technology to supporting exploration missions. To examine its performance from the Sojourner Rover (see Figure 18) a tube used as a guide through which the USDC was inserted and allowed to operate using its own mass as the load. The tube internal diameter was about $3 \mathrm{~mm}$ larger than the external diameter of USDC and the USDC was easily shown to make holes and cores in various rocks (basalt, sandstone, etc.). Also, the device was mounted onto the robotic arm of the FIDO rover (see Figure 19) and drilled with minimal mechanical vibration of the arm and was easily used to reinsert the drill bit into holes that were drilled earlier. The ability of the USDC to drill under low axial load has also made it possible to consider operating from an aerobot or inflatable systems using minimal anchoring.

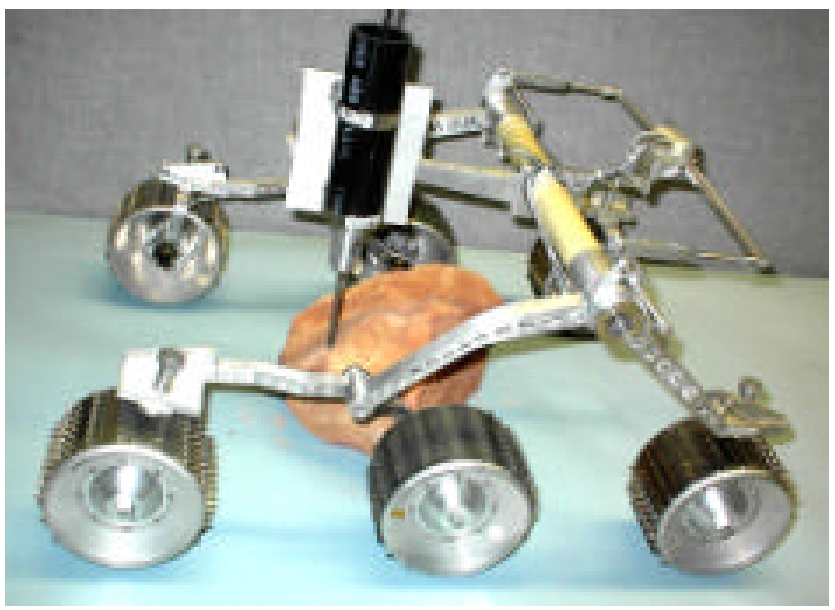

Fig. 18. The USDC mounted on the Sojourner Rover platform is shown to drill sandstone.

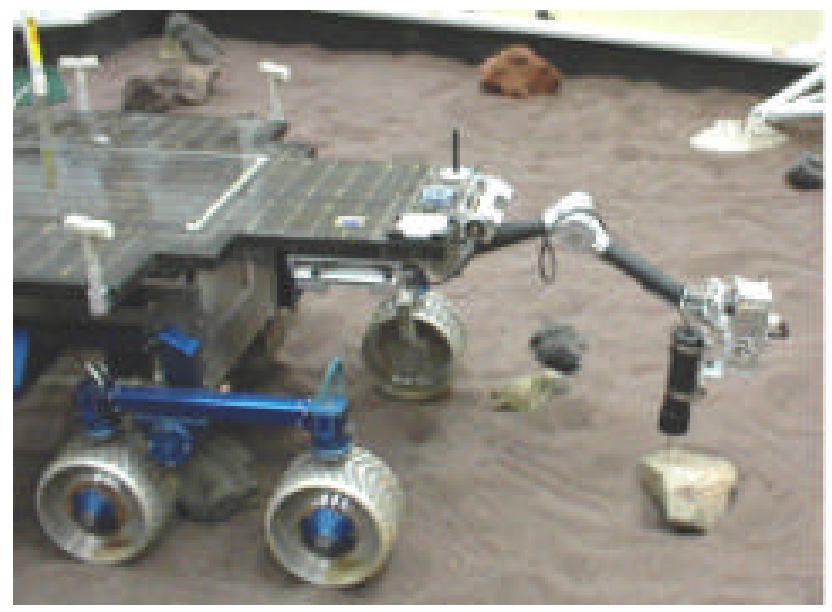

Fig. 19. The USDC is shown drilling while mounted on the robotic arm of the FIDO Rover.

\section{CONCLUSIONS}


A new drilling and coring method was reported allowing operating with low power and needing low axial force. The drill was activated from such platforms as the Sojourner Rover and the robotic arm of the FIDO Rover. By combining resonant elastic vibrations at $20 \mathrm{KHz}$ and inducing a substantial sub-harmonic acoustic component, significant impact force and displacement are generated. The phenomenon is induced by a stack of piezoelectric wafers using an ultrasonic horn and a free-mass that is interfaced with the drill bit. Analytical models were developed to explain the low frequency coupling between the horn, free-mass, drill stem and rock. Current efforts are underway to integrate the various models to allow for the determination of system model with predictive and control abilities. The results suggest a variety of avenues for optimization of the device.

This new drilling and coring device was demonstrated to require a relatively low preload $(<5 \mathrm{~N})$ and can be driven at low power $(5 \mathrm{~W})$. It was used to drill rocks as hard rocks as granite, diorite, basalt and as soft as limestone and sandstone. This USDC has enabled new possibilities in support of future NASA missions including sampling, as well as in-situ probing and analysis. Given the capability of piezoelectric materials a drill can be developed for operation at high or low temperatures allowing sampling at such planet as Venus.

\section{ACKNOWLEDGMENT}

The authors would like to thank J. D. Carson for design and fabrication of the test apparatus. The research at the Jet Propulsion Laboratory (JPL), a division of the California Institute of Technology, was funded by the Mars and Deep Space Exploration Technologies under a contract with the National Aeronautics Space Administration (NASA).

\section{REFERENCES}

[1] W. P. Mason, Electromechanical Transducers and Wave Filters, Princeton, NJ, Van Nostrand, 1948.

[2] W. P. Mason, Physical Acoustics and the Properties of Solids, D. Van Nostrand Co., Princeton, NJ, 1958.

[3] S. Sherrit, B. P. Dolgin, Y. Bar-Cohen, D. Pal, J. Kroh, T. Peterson "Modeling of Horns for Sonic/Ultrasonic Applications," Proceedings of the IEEE International Ultrasonics Symposium, Lake Tahoe, CA, October 1999, pp. 647-651.

\section{BIOGRAPHY}

Dr. Yoseph Bar-Cohen received his M.Sc. in Materials Science in 1973 and his Ph.D. in Physics in 1979 from the Hebrew University in Jerusalem, Israel. $\mathrm{He}$ initiated the development of several innovative devices and mechanisms that are driven by elastic waves and electroactive materials. He

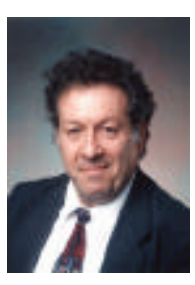
initiated and chairs the SPIE Electroactive Polymers Actuators and Devices Conference and several forums of worldwide EAP technical collaboration, including a newsletter, Web hub and newsgroup. Dr. Bar-Cohen also pioneered several experimental ultrasonic techniques, including the discovery of leaky Lamb waves (LLW) and polar backscattering in composite materials, and he codeveloped related NDE methods. He is an Adjunct Professor at UCLA, a fellow of the American Society of NonDestructive Testing (ASNT), and author/co-author of about 195 publications. He has made numerous presentations at national and international symposia and holds many patents. 